\title{
On the Linearization of Second-Order Differential and Difference Equations
}

\author{
Vladimir DORODNITSYN
}

Keldysh Institute of Applied Mathematics of Russian Academy of Science, 4 Miusskaya Sq., Moscow, 125047 Russia

E-mail:dorod@spp.keldysh.ru

Received November 28, 2005, in final form July 13, 2006; Published online August 16, 2006

Original article is available at http://www.emis.de/journals/SIGMA/2006/Paper065/

\begin{abstract}
This article complements recent results of the papers [J. Math. Phys. 41 (2000), $480 ; 45$ (2004), 336] on the symmetry classification of second-order ordinary difference equations and meshes, as well as the Lagrangian formalism and Noether-type integration technique. It turned out that there exist nonlinear superposition principles for solutions of special second-order ordinary difference equations which possess Lie group symmetries. This superposition springs from the linearization of second-order ordinary difference equations by means of non-point transformations which act simultaneously on equations and meshes. These transformations become some sort of contact transformations in the continuous limit.
\end{abstract}

Key words: non-point transformations; second-order ordinary differential and difference equations; linearization; superposition principle

2000 Mathematics Subject Classification: 34C14; 34C20; 39A05; 65L12; 70H33

\section{Introduction}

The recent article [1] was devoted to symmetry classification of second-order ordinary difference equations. This was based on the paper by S. Lie [3], in which he has provided a symmetry classification of second-order differential equations (ODEs). A special class of the specified invariant second-order difference equations [1] converges to Lie's invariant second-order ordinary differential equations in the continuous limit. The Lagrangian formalism for Lie's list of those second-order ordinary difference equations associated with special invariant meshes (lattices) was developed in recent paper [2]. From the Noether theorem [2] it is known that invariance of a variational functional with respect to an $r$-parameter group leads to existence of $r$ conservation laws for the corresponding Euler's equation. It was shown in [2] (see also [5, 6] for details) that there exists a similar (although more complicated) construction for difference models. This discrete analog of the Noether theorem can be applied to analytic integration of difference equations. Namely, two-dimensional symmetries of the Lagrangian for a second-order difference equation provide two first difference integrals of the two-point type. This yields a general solution of the difference system by purely algebraic manipulations. The last point is important for difference equations since there are no analytic integration techniques for constructing solutions.

The problem of linearization of a given equation (or a system of equations) is of permanent interest in mathematics. For example, in the paper [7] necessary and sufficient conditions, under which a given nonlinear system of PDEs with $n \geq 2$ independent variables and $m \geq 1$ dependent variables can be transformed to a linear system of PDEs, were developed. It was shown that in all the cases of such mappings, an infinite-parameter Lie group of point or contact transformations admitted by the original nonlinear system is needed. 
For ODEs the situation is different. S. Lie proved that symmetry algebras admitted by second-order ODEs are finite, and the maximum symmetry algebra is 8-dimensional. This maximum symmetry algebra is admitted by a linear equation. S.Lie has developed necessary and sufficient conditions under which a given nonlinear second-order ODE can be transformed into a linear one [8] (the discussion of this question can be found, for example, in [9, p. 38]. One of these (equivalent to each other) conditions is existence of two linearly connected symmetry operators. In this case there exists a change of variables that transforms a given nonlinear equation into a linear one. Generalization of this idea to ordinary difference equations was developed in [10] where, for some class of ordinary difference equations on fixed uniform lattice, point transformations which linearize corresponding nonlinear difference equations have been found. This class of ordinary difference equations was restricted by those equations that possess point autonomous symmetries, which do not change the independent variable. Difference equations are assumed to be defined on a fixed regular lattice with unit spacing. This approach was generalized in [11] to non-autonomous symmetries, while the independent variable was still fixed. The discrete analog of Lie's theorem [8], providing the necessary and sufficient conditions, under which a given nonlinear second-order difference equation and a mesh can be transformed into a linear one, was developed in [1, Theorem 1]. It is important to notice that in all the cases the corresponding point transformations change equations within one class of equivalent difference equations, which are invariant under the actions of isomorphic symmetry groups. Point transformations of such type cannot connect nonlinear equations from Lie's list with a linear one. Being based on listing of groups, the symmetry classification of second-order differential [3] and difference equations [1] singled out classes of equations, which cannot be connected by means of point transformations (of independent and dependent variables) and, in particular, cannot be linearized by a point transformation in principle. In the classification [3] S. Lie singled second-order ODEs up to arbitrary point transformations. For example, a linear equation is the simplest representative equation from the class of equivalent equations which possess an 8-dimensional symmetry group.

Thus, in this article we are dealing with "linearization of not-linearizable equations" in the above mentioned sense. We establish non-point transformations connecting some nonlinear invariant second-order differential equations from Lie's list with linear equations. Such transformations have discrete counterparts, and so the invariant difference schemes can be linearized together with difference meshes.

We will use the following notation. Let $x$ be the independent variable and $y$ the dependent variable. To consider a second-order difference equation and a lattice in $x$-direction we need 3-points of a difference mesh (lattice): $\left(x, x_{-}, x_{+}\right)$and corresponding values of the dependent variable in three neighboring points: $\left(y, y_{-}, y_{+}\right)$, where "+" marks a point shifted to the right, and "-" marks a point shifted to the left. We call this set of points (a subspace) $\left(x, x_{-}, x_{+}, y, y_{-}, y_{+}\right)$a difference stencil. It is supposed that the stencil and the corresponding difference equation can be shifted to any other point of the lattice.

The discrete model for second order ODE can be presented in terms of two difference equations:

$$
F\left(x, x_{-}, x_{+}, y, y_{-}, y_{+}\right)=0, \quad \Omega\left(x, x_{-}, x_{+}, y, y_{-}, y_{+}\right)=0
$$

We will also use the following notation for the right and for the left difference derivatives of the first order:

$$
y_{x}=\frac{y_{+}-y}{h_{+}}, \quad y_{\bar{x}}=\frac{y-y_{-}}{h_{-}} .
$$

where $h_{+}, h_{-}$are the space steps of the mesh in the $x$-direction. 
To distinguish between continuous derivatives and difference ones we denote the first by $y^{\prime}, y^{\prime \prime}, \ldots$. The first equation of the system (1) represents a second-order difference equation. In the continuous limit it should become a second-order ordinary differential equation on some point of a finite-dimensional space of continuous variables. The second equation yields a lattice (mesh) on which the first equation is considered. Notice that the mesh equation does not need to be a second-order differential equation in the continuous limit. For example, one can consider a uniform lattice equation $h_{+}=h_{-}$(where $\left.h_{+}=x_{+}-x, h_{-}=x-x_{-}\right)$which "disappears" in the continuous limit.

The group transformations considered in the present approach are of the same type as for ODEs. They are generated by a Lie algebra of vector fields of the form

$$
X=\xi(x, y) \frac{\partial}{\partial x}+\eta(x, y) \frac{\partial}{\partial y} .
$$

The corresponding transformations are purely point ones, since the coefficients $\xi$ and $\eta$ depend on $(x, y)$ only, and do not depend on the shifted points $\left(x_{+}, y_{+}\right)$or $\left(x_{-}, y_{-}\right)$.

In the classification of second-order ODEs [3] S. Lie identified a linear equation, which possess 8-dimensional symmetry group; four simplest representative nonlinear equations which possess a 3-dimensional symmetry group; 2 classes of ODEs with a 2-dimensional symmetry group and one class of ODEs with a one-dimensional symmetry group.

In the following sections we consider three examples of ODEs that possess three symmetries. In parallel we will consider the corresponding difference models as developed in [2]. Also we consider examples from the Lie classes of nonlinear ODEs with a two-dimensional Lie algebra. The chosen equations are important from the physical point of view.

We will write down explicitly the linearizing tangent transformations for underlining secondorder nonlinear ODEs. We will also write down explicitly the linearizing two-points transformations for corresponding difference equations and meshes. Notice that these non-point transformations change the admitted symmetries of the underlying equations as the corresponding linear equations possess the 8-dimensional symmetry. In fact, these transformations are not explicitly connected with admitted symmetries of ODEs. This aspect will be discussed later.

Notice that all difference and differential equations considered here are integrable (at least in quadratures) as far as they possess a symmetry algebra of dimension not less than 2 . Therefore, the computational aspects of the invariant difference schemes (as well as stability, convergence of developed schemes, etc.) will not discussed here.

In the present paper we are following the set of invariant ODEs for which an appropriate invariant difference equations and meshes were developed in [1, 2].

It seems that the main result presented below is the explicit non-point transformations, which linearize second-order ODEs from the Lie's list and appropriate invariant difference equations, which possess symmetries of the dimension less than 8. A discussion of the origin of such transformations will be presented at the end of Example 1 and in Concluding remarks.

\section{Example 1: ODE with a 3-dimensional symmetry group}

We start with the example of a second-order nonlinear ODE from the Lie's list of equations, which possesses three symmetries. It has two first integrals and, therefore, can be integrated by means of algebraic manipulations with difference integrals.

The group given by the operators

$$
X_{1}=\frac{\partial}{\partial x}, \quad X_{2}=2 x \frac{\partial}{\partial x}+y \frac{\partial}{\partial y}, \quad X_{3}=x^{2} \frac{\partial}{\partial x}+x y \frac{\partial}{\partial y}
$$


corresponds to the invariant differential equation from the Lie's list,

$$
y^{\prime \prime}=y^{-3} \text {. }
$$

This equation can be obtained from a variational functional with the Lagrangian function $L=y^{\prime 2}-\frac{1}{y^{2}}$ which admits all three operators (2) as variational symmetries.

The Noether theorem [4] yields the following first integrals:

$$
J_{1}=y^{\prime 2}+\frac{1}{y^{2}}=A_{0}, \quad J_{2}=\frac{x}{y^{2}}-\left(y-y^{\prime} x\right) y^{\prime}=B_{0} .
$$

By means of the first integrals we write the general solution $y(x)$ as

$$
A_{0} y^{2}=\left(A_{0} x-B_{0}\right)^{2}+1 .
$$

Now we reproduce the procedure of the linearization for the equation (3), followed by explanatory comments.

It turns out that equation (3) can be linearized by the non-point transformation

$$
t=x\left(y^{\prime 2}+\frac{1}{y^{2}}\right)+\arctan \left(y y^{\prime}\right)-y y^{\prime}, \quad y(x)=\frac{1}{u(t)}, \quad y^{\prime}=-u^{\prime},
$$

which transforms equation (3) into the following linear equation

$$
u^{\prime \prime}+u=0,
$$

which possesses a linear superposition for its solutions.

Thus, the developed formulas implicitly reproduce the nonlinear superposition principle for equation (3). The transformation (6) changes the first integrals (4) into the following ones with the same constants $A_{0}, B_{0}$ :

$$
u^{\prime 2}+u^{2}=A_{0}, \quad t+\arctan \left(\frac{u^{\prime}}{u}\right)=B_{0} .
$$

The latter yields the general solution of the linear equation (7)

$$
u(t)=\sqrt{A_{0}} \cos \left(B_{0}-t\right)=\tilde{A} \sin t+\tilde{B} \cos t .
$$

Notice that (6) connects the general solution (5) with (10), which provides an explicit procedure for the superposition of solutions (5). Indeed, let us take two solutions among the family (5), which correspond to the two sets of constants: $y_{1}=Y_{1}\left(x, A_{1}, B_{1}\right)$ and $y_{2}=Y_{2}\left(x, A_{2}, B_{2}\right)$. Then the transformation (6) produces two solutions of the linear equation (7),

$$
u_{1}(t)=\sqrt{A_{1}} \cos \left(B_{1}-t\right), \quad u_{2}(t)=\sqrt{A_{2}} \cos \left(B_{2}-t\right) .
$$

The sum of these solutions

$$
\begin{aligned}
& u(t)=u_{1}+u_{2}=\sqrt{A^{*}} \cos \left(B^{*}-t\right), \\
& A^{*}=A_{1}+A_{2}+2 \sqrt{A_{1} A_{2}} \cos \left(B_{1}-B_{2}\right), \quad B^{*}=\arctan \frac{\sqrt{A_{1}} \sin B_{1}+\sqrt{A_{2}} \sin B_{2}}{\sqrt{A_{1}} \cos B_{1}+\sqrt{A_{2}} \cos B_{2}},
\end{aligned}
$$

yields a new solution of equation (3) with two constants $A^{*}, B^{*}$. The developed formulas reproduce the nonlinear superposition principle within the family of solutions (5). For example, the solutions $y_{1}=\sqrt{x^{2}+1}$ and $y_{2}=\sqrt{4 x^{2}+\frac{1}{4}}$ produce the new one $y_{3}=\sqrt{7 x^{2}+\frac{1}{7}}$. 
Now we consider the discrete case. In the paper [2] a difference scheme which preserves symmetries and the Lagrangian structure of the initial equation (3) was developed.

The starting point was an entire set of finite-difference invariants

$$
I_{1}=\frac{h_{+}}{y y_{+}}, \quad I_{2}=\frac{h_{-}}{y y_{-}}, \quad I_{3}=\frac{y^{2} y_{-}}{h_{-}}\left(\frac{y_{+}-y}{h_{+}}-\frac{y-y_{-}}{h_{-}}\right) .
$$

in a subspace of difference variables (difference stencil) $x, h_{+}, h_{-}, y, y_{+}, y_{-}$.

Any difference equation which can be represented by means of the above invariants will be invariant. Thus, in such a way one can generate an invariant mesh. We will use the following relation between difference invariants

$$
\frac{h_{+}}{y y_{+}}=\frac{h_{-}}{y y_{-}}
$$

as the invariant mesh (see [2]).

It is easy to see that the mesh equation has a first integral

$$
\frac{h_{+}}{y y_{+}}=\varepsilon, \quad \varepsilon=\text { const. }
$$

As an invariant representation of the equation (3) we consider the following:

$$
\frac{1}{h_{-}}\left(\frac{y_{+}-y}{h_{+}}-\frac{y-y_{-}}{h_{-}}\right)=\frac{1}{y^{2} y_{-}} \text {. }
$$

The last equation on the mesh (8) can be rewritten as the mapping

$$
y_{+} y\left(2-\varepsilon^{2}\right)=y\left(y_{+}+y_{-}\right) .
$$

Notice that difference model (8), (9) of equation (3) can be developed from the discrete Lagrangian function $\mathcal{L}=y_{x}^{2}-\frac{1}{y y^{+}}$which gives first integrals by means of a finite-difference analog of the Noether theorem [5, 6]:

$$
I_{1}=y_{x}^{2}+\frac{1}{y y^{+}}=A, \quad I_{2}=\frac{x+x^{+}}{2 y y^{+}+y_{x}\left(x^{+} y_{x}-y^{+}\right)}=B .
$$

Eliminating $y_{x}, x_{+}$and $y_{+}$from the first difference integrals, we obtain the general solution

$$
A y^{2}=(A x-B)^{2}+1-\frac{\varepsilon^{2}}{4},
$$

which agrees with the continuous limit up to order $\varepsilon^{2}$.

To obtain the difference analog of the non-point transformation (6) we rewrite the latter as relations between the differentials:

$$
d x=\frac{d t}{u^{2}}, \quad y(x)=\frac{1}{u(t)} .
$$

Then, as a difference analog of the above transformation, we will use non-point transformations for discrete variables as follows

$$
y=\frac{1}{u}, \quad y^{+}=\frac{1}{u^{+}}, \quad y^{-}=\frac{1}{u^{-}}, \quad \tau^{+}=\frac{h^{+}}{y y^{+}}, \quad \tau^{-}=\frac{h^{-}}{y y^{-}}, \quad y_{x}=-u_{t} .
$$


where $\tau^{+}$and $\tau^{-}$are the mesh steps in the new coordinate system. Invariant mesh (8) is transformed into the following regular mesh in the new coordinate system:

$$
\tau^{+}=\tau^{-}=\tau_{0}=\varepsilon,
$$

and the nonlinear equation (9) becomes linear:

$$
\frac{u^{+}-2 u+u^{-}}{\tau_{0}^{2}}+u=0 .
$$

The general solution of the linear equation is

$$
u(t)=\sqrt{A_{0}} \cos \left(B_{0}-\frac{\phi}{\tau_{0}} t\right), \quad \phi=\arccos \left(\frac{2-\tau_{0}^{2}}{2}\right) .
$$

The transformation (12) changes the first integrals (10) into the following ones:

$$
u_{t}^{2}+u u^{+}=A_{0}, \quad\left(\frac{\phi}{\tau_{0}} t+\frac{\phi}{2}\right)-\frac{1}{2} \arccos \left(\frac{2}{\left(1+\frac{u_{t}^{2}}{u u^{+}}\right)}-\cos \phi\right)=B_{0} .
$$

The solution family (11), as well as the family (13), are quite similar to those of the corresponding differential equations, and the superposition principle for two solutions of the difference scheme (8), (9) can be formulated in the same manner as for the continuous case.

Notice that the linearizing transformations presented above change the admitted symmetries of the underlining equation. The way to obtain such transformation could be viewed as follows. The first step is construction of an invariant difference model (that was done in [1,2]) with the conservation of Lagrangian structure, to make the Noether-type theorems applicable. Then, as the second step, we find a difference non-point transformation, which makes the nonlinear invariant mesh regular (since otherwise even linear difference equation would be nonlinear). This step is quite evident from the structure of the mesh equation. The third step is to obtain a continuous limit of the transformation of lattice, and then the corresponding tangent transformations in the space $x, y, t, u$, which preserve both constants of the two first integrals. As a last step, we complete the appropriate linearizing transformations for discrete models.

It should be pointed out that the linearizing transformations are not unique. Indeed, one can find another relation between first integrals as far as any integral can be expressed by means of any smooth function of two independent first integrals. We should also add that existence of two explicit first integrals allows one to express the linearizing transformation explicitly from the space $\left(x, y, y^{\prime}\right)$ to the space $\left(t, u, u^{\prime}\right)$. In Example 4 we will deal with a generalization of equation (3), which has two-dimensional symmetry and the only first integral. In that case we are able to construct linearizing transformations as connections between differentials.

In the following examples we briefly reproduce the linearizing transformations for both invariant ODEs and corresponding invariant difference equations and meshes.

\section{Example 2: ODE with 3-dimensional symmetry group}

The differential equation

$$
y^{\prime \prime}=\left(y^{\prime}\right)^{\frac{k-2}{k-1}}, \quad k \neq 1
$$

has a symmetry algebra generated by the following operators

$$
X_{1}=\frac{\partial}{\partial x}, \quad X_{2}=\frac{\partial}{\partial y}, \quad X_{3}=x \frac{\partial}{\partial x}+k y \frac{\partial}{\partial y}, \quad k \neq 0, \frac{1}{2}, 1,2 .
$$


This equation can be obtained by the variational procedure from the Lagrangian

$$
L=\frac{(k-1)^{2}}{k}\left(y^{\prime}\right)^{\frac{k}{k-1}}+y
$$

which admits operators $X_{1}$ and $X_{2}$ for any parameter $k \neq 1$, and therefore the Noether theorem yields two first integrals

$$
J_{1}=\frac{(1-k)}{k}\left(y^{\prime}\right)^{\frac{k}{k-1}}+y=A^{0}, \quad J_{2}=(k-1)\left(y^{\prime}\right)^{\frac{1}{k-1}}-x=B^{0} .
$$

Eliminating $y^{\prime}$ we find the general solution:

$$
y=\frac{1}{k}\left(\frac{1}{k-1}\right)^{(k-1)}\left(x+B^{0}\right)^{k}+A^{0} .
$$

Following the same procedure as described in Example 1, one can find the non-point transformation

$$
x=-V(t), \quad y=t+\frac{k-1}{k}\left(V^{\prime}\right)^{k}+(k-1) \ln V^{\prime}, \quad y^{\prime}=\left(V^{\prime}\right)^{k-1},
$$

which transforms equation (14) into the following linear equation

$$
(k-1) V^{\prime \prime}+V^{\prime}=0 .
$$

The transformation (15) changes the first integrals into the following ones

$$
(k-1) \ln V^{\prime}+t=A_{0}, \quad(k-1) V^{\prime}+V=B_{0} .
$$

The latter yields the general solution of the linear equation (16):

$$
V(t)=(1-k) e^{\frac{A_{0}-t}{k-1}}+B_{0} .
$$

As an invariant difference scheme for the equation (14), the following difference equations were constructed [2]:

$$
\frac{2 \alpha(k-1)}{h_{+}+h_{-}}\left(\left(y_{x}\right)^{\frac{1}{k-1}}-\left(y_{x}^{-}\right)^{\frac{1}{k-1}}\right)=1, \quad \frac{h_{+}}{\left(y_{x}\right)^{\frac{1}{k-1}}}=\frac{h_{-}}{\left(y_{x}^{-}\right)^{\frac{1}{k-1}}},
$$

where the constant $\alpha$ should be chosen in accordance with the compatibility condition for the first integrals (see [2] for details).

We again rewrite the transformation (15) as a connection between differentials

$$
d t=\frac{d x}{\left(y^{\prime}\right)^{\frac{1}{k-1}}}, \quad\left(y^{\prime}\right)^{\frac{1}{k-1}}=-V^{\prime}
$$

and then involve transformations as a two-point change of variables

$$
\left(y_{x}\right)^{\frac{1}{k-1}}=-V_{t}, \quad\left(y_{x}^{-}\right)^{\frac{1}{k-1}}=-V_{t}^{-}, \quad \tau^{+}=\frac{h_{+}}{\left(y_{x}\right)^{\frac{1}{k-1}}}, \quad \tau^{-}=\frac{h_{-}}{\left(y_{x}^{-}\right)^{\frac{1}{k-1}}} .
$$

Evidently, the transformed mesh will be regular

$$
\tau^{+}=\tau^{-}=\tau^{0},
$$

and the equation will be linear

$$
2 \alpha(k-1) \frac{V_{t}-V_{t}^{-}}{\tau^{0}}+\frac{V_{t}+V_{t}^{-}}{2}=0 .
$$




\section{Example 3: ODE with a 3-dimensional symmetry group}

We consider one more three-dimensional group and its Lie algebra,

$$
X_{1}=\frac{\partial}{\partial x}, \quad X_{2}=\frac{\partial}{\partial y}, \quad X_{3}=x \frac{\partial}{\partial x}+(x+y) \frac{\partial}{\partial y} .
$$

The corresponding invariant second-order ODE is the following:

$$
y^{\prime \prime}=\exp \left(-y^{\prime}\right) \text {. }
$$

It can be obtained from the Lagrangian function

$$
L=\exp \left(y^{\prime}\right)+y
$$

which is divergently invariant with respect to $X_{1}, X_{2}$. The corresponding first integrals of the equation (17) are

$$
\exp \left(y^{\prime}\right)-x=A_{0}, \quad \exp \left(y^{\prime}\right)\left(1-y^{\prime}\right)+y=B_{0} .
$$

So, the general solution of equation (17) is

$$
y=\left(x+B_{0}\right)\left(\ln \left(x+B_{0}\right)-1\right)+A_{0} .
$$

The equation (17) can be linearized by the non-point transformation

$$
x=V(t), \quad y=\left(1+V^{\prime}\right) \ln V^{\prime}-V^{\prime}-t, \quad y^{\prime}=\ln V^{\prime},
$$

which transforms the equation (17) into the following linear one

$$
V^{\prime \prime}-V^{\prime}=0
$$

The transformation (19) changes the first integrals (18) into

$$
V^{\prime}-V=A_{0}, \quad \ln V^{\prime}-t=B_{0},
$$

which yield the general solution of the linear equation,

$$
V(t)=e^{B_{0}+t}-A_{0} .
$$

The superposition principle for solutions of (17) can be established similarly to Example 1.

The invariant difference model constructed in [2] is the following:

$$
\begin{aligned}
& \frac{\beta}{h_{+}}\left(e^{y_{x}}-e^{y_{x}^{-}}\right)=1, \\
& \frac{h_{+}}{e^{y_{x}}}=\frac{h_{-}}{e^{y_{x}^{-}}},
\end{aligned}
$$

where

$$
\beta=\frac{e^{1+\varepsilon^{2}}}{(1+\varepsilon)^{1+\frac{1}{\varepsilon}}}, \quad \frac{h_{+}}{e^{y_{x}}}=\varepsilon=\text { const }>0,
$$

the constants $\beta, \varepsilon$ are chosen in accordance with the compatibility condition for the first integrals. 
The above difference model preserves both all symmetries of the original differential equation and the Lagrangian structure (see [2]), and therefore can be integrated. The general solution is

$$
y=\left(x+B_{0}\right) \ln \left(x+B_{0}\right)-\left(1+\varepsilon^{2}\right)\left(x+B_{0}\right)+A_{0}, \quad h_{+}=\varepsilon\left(x+B_{0}\right) .
$$

This difference model can also be linearized. We rewrite the transformation (19) as

$$
V^{\prime}=e^{y^{\prime}}, \quad d t=\frac{d x}{e^{y^{\prime}}},
$$

and find the corresponding difference transformations as

$$
V_{t}=e^{y_{x}}, \quad V_{t}^{-}=e^{y_{x}^{-}}, \quad \tau^{+}=\frac{h^{+}}{e^{y_{x}}}, \quad \tau^{-}=\frac{h^{-}}{e^{y_{x}^{-}}} .
$$

Applying the above transformation we linearize the difference equation (20) together with the mesh (21),

$$
\frac{V^{+}-2 V+V^{-}}{\tau_{0}^{2}}-\frac{V^{+}-V^{-}}{2 \tau_{0}}=0, \quad \tau^{+}=\tau^{-}=\tau_{0} .
$$

The last fourth Lie's equation, which possesses 3-dimensional symmetry, contains an arbitrary constant, and it is a first integral of some nonlinear third-order ODE (the differential consequence of this second-order ODE). The linearization of this equation is rather complicated and is not considered here.

\section{Example 4: ODE with a 2-dimensional symmetry group}

S. Lie identified two classes of ODEs which possess a 2-dimensional symmetry group. Both classes contain arbitrary functions. For example, one class of equations is the following

$$
y^{\prime \prime}=\frac{1}{x} F\left(y^{\prime}\right),
$$

which is invariant with respect to the group with the following operators

$$
X_{1}=\frac{\partial}{\partial y}, \quad X_{2}=x \frac{\partial}{\partial x}+y \frac{\partial}{\partial y} .
$$

It seems to be impossible to linearize the whole class of invariant ODEs, thus we consider just representative equations. We will choose the example which is important from the physical point of view.

First, we interchange dependent and independent variables and, second, we make scaling of the independent variable to obtain another representation of the underlining class of equations:

$$
y^{\prime \prime}=y^{n} F\left(x^{2} y^{n-1}\right),
$$

where $n$ is some constant, $n \neq-1$.

Now we consider the special case of the above equation, when the function $F=1$. It will be the general case of the power nonlinearity, i.e. the differential equation

$$
y^{\prime \prime}=y^{n}, \quad n \neq-1,
$$

which possesses an isomorphic two-dimensional Lie algebra of operators

$$
X_{1}=\frac{\partial}{\partial x}, \quad X_{2}=(n-1) x \frac{\partial}{\partial x}-2 y \frac{\partial}{\partial y} .
$$


Notice, that this example makes sense from the physical point of view, as equation (22) can be considered as a dynamical equation for a particle in a field of the power potential. This equation can be obtained from the Lagrangian function

$$
L=y^{\prime 2}+\frac{2}{n+1} y^{n+1},
$$

which admits the only operator $X_{1}$.

In contrast to Example 1, which is a special case of equation (22) with $n=-3$, the underlining equation for an arbitrary $n$ has a unique first integral:

$$
J_{1}=y^{\prime 2}-\frac{2}{n+1} y^{n+1}=A^{0} .
$$

The second integral can be expressed by a quadrature

$$
x-\int \frac{d y}{\sqrt{A_{0}+\frac{2}{n+1} y^{n+1}}}=B_{0},
$$

and for the only cases

$$
n=\frac{1}{k}-1, \quad n=\frac{1-2 k}{1+2 k},
$$

where $k$ is integer, this integral can be expressed by a finite superposition of elementary functions. We see that equation (3), considered in Example 1, corresponds to $k=-1$. In contrast to a general case, equation (3) is the only equation of power nonlinearity which possesses a 3dimensional symmetry group. The exceptional potential which corresponds to equation (3) is known in theoretical physics.

Thus, the example of the general power potential under consideration is sufficiently different from the above three, as we have fewer symmetries and the only first explicit integral. Nevertheless, equation (22) for any $n$ can be linearized by a non-point transformation. The only disadvantage is that we will express the linearizing transformation in terms of differentials.

Again, we would like to preserve the first integral under linearization, for example, as follows:

$$
y^{\prime 2}-\frac{2}{n+1} y^{n+1}=A^{0}=u^{\prime 2}-u^{2} .
$$

Then, we can choose the change of dependent variables and derivatives

$$
\frac{2}{n+1} y^{n+1}=u^{2}, \quad y^{\prime}(x)=u^{\prime}(t)
$$

and, therefore, the transformation of differentials and derivatives could be the following

$$
d x=\left|\frac{n+1}{2}\right|^{-\frac{n}{n+1}} u^{\frac{1-n}{n+1}} d t, \quad y(x)=\left|\frac{n+1}{2}\right|^{\frac{1}{n+1}} u^{\frac{2}{n+1}}, \quad y^{\prime}(x)=u^{\prime}(t) .
$$

The transformation (24) transforms the equation (22) into the following linear one

$$
u^{\prime \prime}-u=0
$$

which has the first integral

$$
u^{\prime 2}-u^{2}=A_{0} .
$$


Meanwhile the equation (25) has one more functionally independent integral

$$
\left(u^{\prime}+u\right) e^{-t}=B_{0},
$$

and therefore the general solution is

$$
u(t)=A e^{t}+B e^{-t} .
$$

Notice that one can transform backwards the above general solution and the second integral just for exceptional values of $n$.

We will construct an invariant difference model by means of full set of difference invariants of the Lie algebra (23) on a difference stencil:

$$
I_{1}=\frac{h_{+}}{h_{-}}, \quad I_{2}=\frac{y_{+}}{y}, \quad I_{3}=\frac{y_{-}}{y}, \quad I_{4}=h_{+} y_{+} \frac{n-1}{4} y^{\frac{n-1}{4}} .
$$

As an invariant difference mesh, we will use the following relation between difference invariants:

$$
h_{+} y_{+} \frac{n-1}{4}=h_{-} y_{-} \frac{n-1}{4},
$$

which has the integral

$$
h_{+} y_{+} \frac{n-1}{4} y^{\frac{n-1}{4}}=\varepsilon, \quad \varepsilon=\text { const. }
$$

By means of difference invariants (26) one can develop an invariant approximation of equation (22) on mesh (27):

$$
\left(\frac{y_{+}^{\frac{n+1}{2}}-y^{\frac{n+1}{2}}}{h_{+} y_{+} \frac{n-1}{4}}-\frac{y^{\frac{n+1}{2}}-y_{-}^{\frac{n+1}{2}}}{h_{-} y_{-}^{\frac{n-1}{4}}}\right) \frac{1}{h_{+}}=y_{+}^{\frac{n-1}{4}} y^{n} .
$$

As the difference analog of the transformation (24), we will use the non-point transformations for discrete variables as follows

$$
\begin{array}{ll}
u(t)=\left|\frac{n+1}{2}\right|^{-\frac{1}{2}} y^{\frac{n+1}{2}}, & u^{+}=\left|\frac{n+1}{2}\right|^{-\frac{1}{2}} y_{+}^{\frac{n+1}{2}}, \quad u^{-}=\left|\frac{n+1}{2}\right|^{-\frac{1}{2}} y_{-}^{\frac{n+1}{2}}, \\
\tau^{+}=h^{+} y^{\frac{n-1}{4}} y_{+}^{\frac{n-1}{4}}, & \tau^{-}=h^{-} y^{\frac{n-1}{4}} y_{-}^{\frac{n-1}{4}},
\end{array}
$$

where $\tau^{+}$and $\tau^{-}$are the new mesh steps.

Invariant mesh (27) is transformed into the regular one

$$
\tau^{+}=\tau^{-}=\tau_{0}=\varepsilon,
$$

and the nonlinear equation (28) becomes linear,

$$
\frac{u^{+}-2 u+u^{-}}{\tau_{0}^{2}}-u=0 \text {. }
$$

Equation (29) has the following difference integrals

$$
u_{t}^{2}-u u^{+}=A_{0}, \quad\left(u_{t}+\beta u^{+}\right) q^{\frac{t}{\tau_{0}}}=B_{0},
$$

where

$$
\beta=\frac{\tau_{0}+\sqrt{\tau_{0}^{2}+4}}{2}, \quad q=\frac{-\tau_{0}+\sqrt{\tau_{0}^{2}+4}}{\tau_{0}+\sqrt{\tau_{0}^{2}+4}} .
$$

The general solution of (29) can be found from the above integrals. Alternatively, one can look for a solution in the form

$$
u(t)=A q_{1}{ }^{\frac{t}{\tau_{0}}}+B q_{2}{ }^{\frac{t}{\tau_{0}}} .
$$

Substituting (30) into (29) yields the needed constants

$$
q_{1,2}=\frac{\tau_{0}^{2}+2 \pm \tau_{0} \sqrt{\tau_{0}^{2}+4}}{2} .
$$




\section{Example 5: special case of an ODE with a 2-dimensional symmetry group}

For the special case $n=-1$ the corresponding ODE

$$
y^{\prime \prime}=y^{-1},
$$

possesses the two-dimensional Lie algebra given by the operators

$$
X_{1}=\frac{\partial}{\partial x}, \quad X_{2}=x \frac{\partial}{\partial x}+y \frac{\partial}{\partial y} .
$$

Equation (31) can be obtained as the Euler equation from the Lagrangian

$$
L=\ln y+\frac{1}{2}\left(y^{\prime}\right)^{2}
$$

which is invariant with respect to $X_{1}$.

The corresponding first integral of equation (31) is

$$
\ln y-\frac{1}{2}\left(y^{\prime}\right)^{2}=A_{0},
$$

while the second integral could be expressed by a quadrature

$$
x-\int \frac{d y}{\sqrt{A_{0}+2 \ln y}}=B_{0} .
$$

Following the way of preservation of the first integral under the linearizing transformations

$$
\ln y-\frac{1}{2}\left(y^{\prime}\right)^{2}=A_{0}=u-\frac{1}{2}\left(u^{\prime}\right)^{2},
$$

we choose the change of dependent variable and derivative as follows

$$
\ln y(x)=u(t), \quad y^{\prime}(x)=u^{\prime}(t) .
$$

Then the independent variables are connected as

$$
d x=e^{u} d t .
$$

It follows from first integral (33) that the second-order ODE is the following:

$$
u^{\prime \prime}=1 \text {. }
$$

Thus, equation (31) can be linearized by a non-point transformation

$$
y(x)=e^{u(t)}, \quad d x=e^{u} d t, \quad y^{\prime}=u^{\prime},
$$

which transforms equation (31) into the linear ODE (34).

The transformation (35) changes the first integral (32) into the following:

$$
u-\frac{1}{2}\left(u^{\prime}\right)^{2}=A_{0} .
$$

For the linear equation (34) there exists one more independent first integral

$$
u^{\prime}-t=B_{0},
$$


which yields the general solution of the linear equation in the form

$$
u(t)=\frac{\left(t+B_{0}\right)^{2}}{2}+A_{0}
$$

Thus, the linearizing transformation (35) yields an implicit superposition principle for equation $(31)$.

An invariant difference model can be obtained from the following difference Lagrangian:

$$
L=\ln y+\ln y^{+}+\left(y_{x}\right)^{2},
$$

where $y_{x}=\frac{y^{+}-y}{h_{+}}$is a right difference derivative.

The application of the difference analog of the Noether theorem [5] yields the appropriate quasi-extremal equation

$$
y_{x}-y_{x}{ }^{-}=\frac{\ln y^{+}-\ln y^{-}}{y_{x}+y_{x}^{-}},
$$

where $y_{x}{ }^{-}=\frac{y-y^{-}}{h_{-}}$is a left difference derivative, and the first integral

$$
\ln \left(y^{+} y\right)-y_{x}^{2}=2 A .
$$

The invariant difference mesh for (37) should be expressed by means of difference invariants

$$
h^{+}=h^{-} F\left(\frac{y^{+}}{y}, \frac{y}{y^{-}}, \frac{h^{-}}{y}\right),
$$

and it is not yet defined.

As a difference model for (34) we consider the following linear second-order equation

$$
\frac{u^{+}-2 u+u^{-}}{\tau^{+}}=\frac{1}{\tau^{+}}\left(u_{t}-u_{t}^{-}\right)=1,
$$

on regular lattice

$$
\tau^{+}=\tau^{-} .
$$

The last model has the following first integrals:

$$
\begin{aligned}
& \left(u^{+}+u\right)-u_{t}^{2}=2 A, \\
& u_{t}-\left(t+\frac{\tau^{+}}{2}\right)=B,
\end{aligned}
$$

and mesh equation (39) has an evident integral

$$
\tau^{+}=\tau^{-}=\tau_{0}=\text { const. }
$$

From two first integrals (40), (41) one can exclude $u^{+}$and obtain a general solution

$$
u(t)=\frac{(t+B)^{2}}{2}-\frac{\tau_{0}^{2}}{8}+A,
$$

which well corresponds to the solution (36) of ODE (34).

Now we can write down the linearizing transformation

$$
u=\ln y, \quad u^{+}=\ln y^{+}, \quad u^{-}=\ln y^{-}, \quad u_{t}=y_{x}, \quad u_{t}^{-}=y_{x}{ }^{-},
$$




$$
\tau^{+}=\frac{\ln y^{+}-\ln y}{y^{+}-y} h^{+}, \quad \tau^{+}=\frac{\ln y-\ln y^{-}}{y-y^{-}} h^{-},
$$

which preserves both the first integral (40) of equation (38) and the integral of a mesh. Notice that in the continuous limit transformation (42) tends to (35). Under the action of (42) the regular mesh (39) is transformed into the following non-regular invariant mesh

$$
h^{+} \frac{\ln \frac{y^{+}}{y}}{y^{+}-y}=h^{-} \frac{\ln \frac{y}{y^{-}}}{y-y^{-}},
$$

which completes the difference model for ODE (31).

Thus, invariant model (37), (43) is connected with the linear model (38), (39) by two-points transformation (42).

The remaining class of second-order ODEs from the Lie's list contains all autonomous equations which are invariant with respect to the only operator $X_{1}=\frac{\partial}{\partial x}$. For this class the wellknown procedure reduces the order of equation by one. A similar reduction can be done for any autonomous difference equation on a lattice which is invariant with respect to a translation of $x$.

\section{Concluding remarks}

It was shown that, for all examples of invariant differential equations from the Lie's list, there exist non-point linearizing transformations (which transform the family of solutions of the original nonlinear ODE into the family of solutions of a linear one) and, therefore, nonlinear superposition principle for their solutions.

The procedure that we followed in this article is based on the preservation of at least one first integral for both the ODE and the difference equation, and a transformation of the invariant nonlinear difference mesh into a regular one. We started from the continuous case, where second-order ODEs possessing symmetry were already known. From $[1,2]$ we know the symmetry preserving the difference model, i.e., the difference equation and a mesh, which in addition conserves the Lagrangian structure of the original ODE. Then we choose the change of dependent variables which preserves the known first integral. These transformations can be different for different representations of first integrals, and we choose such change of variables, which makes an invariant mesh regular. Then we obtain a continuous limit of these transformations of a lattice and derive the corresponding transformations in the space $(y, d x, u, d t)$, preserving all first integrals. For the cases of two explicit integrals (Examples 1, 2, 3), we express explicitly the change of variables in the space $\left(x, y, y^{\prime}, t, u, u^{\prime}\right)$. Finally, we complete the appropriate linearizing transformations for the discrete model. Notice that the developed non-point linearizing transformations are not contact transformation in the space $\left(x, y, y^{\prime}, t, u, u^{\prime}\right)$, but are such on the family of solutions of invariant ODEs.

It worth mentioning that all difference and differential equations considered here are integrable (at least in quadratures) as far as they possess a symmetry algebra of the dimension not smaller than two. Thus, the integrability is not of interest for these equations. The result is the explicit non-point linearization of integrable nonlinear differential and difference models. The second valuable result of the linearization is a nonlinear superposition principle for solutions of nonlinear equations, which have definite physical meaning. Finally, we can indicate that all mentioned properties can be preserved in finite difference models.

\section{Acknowledgments}

The author thanks P. Winternitz and E. Ferapontov for helpful discussions and remarks. The author's research was sponsored in part by the Russian Fund for Basic Research under the research project No 06-01-00707. 
[1] Dorodnitsyn V., Kozlov R., Winternitz P., Lie group classification of second order difference equations, J. Math. Phys., 2000, V.41, 480-504.

[2] Dorodnitsyn V., Kozlov R., Winternitz P., Continuous symmetries of Lagrangians and exact solutions of discrete equations, J. Math. Phys., 2004, V.45, 336-359, nlin.SI/0307042.

[3] Lie S., Klassifikation und Integration von gewöhnlichen Differentialgleichunden zwischen $x, y$ die eine Gruppe von Transformationen gestatten, Math. Ann., 1888, V.32, 213-281.

[4] Noether E., Invariante Variationsprobleme, Nachr. Konig. Gesell. Wissen., Gottingen, Math.-Phys. Kl., 1918, Heft 2, 235-257.

[5] Dorodnitsyn V., Noether-type theorems for difference equations, Appl. Numer. Math., 2001, V.39, 307-321.

[6] Dorodnitsyn V., The group properties of difference equations, Moscow, Fizmatlit, 2001 (in Russian).

[7] Kumei S., Bluman G.W., When nonlinear differential equations are equivalent to linear differential equations, SIAM J. Appl. Math., 1982, V.42, 1157-1173.

[8] Lie S., Klassifikation und Integration von gewöhnlichen Differentialgleichunden zwischen $x, y$ die eine Gruppe von Transformationen gestatten. I, II, III, IV, Reprinted in Lie's Gessamelte Abhandlungen, Vol. 5, Leipzig, Teubner, 1924.

[9] Ames W.F., Anderson R.L., Dorodnitsyn V.A., Ferapontov E.V., Gazizov R.K., Ibragimov N.H., Svirshchevskii S.R., CRC hand-book of Lie group analysis of differential equations, Vol. I: Symmetries, exact solutions and conservation laws, Editor N.H. Ibragimov, CRC Press, 1994.

[10] Maeda S., The similarity method for difference equations, IMA J. Appl. Math., 1987, V.38, $129-134$.

[11] Byrnes G.B., Sahadevan R., Quispel G.R.W., Factorizable Lie symmetries and the linearization of difference equations, Nonlinearity, 1995, V.8, 44-59. 